\title{
High Stimulatory Activity of Dendritic Cells from Diabetes-prone BioBreeding/ Worcester Rats Exposed to Macrophage-derived Factors
}

\author{
Anna Tafuri, * William E. Bowers, ${ }^{*}$ Eugene S. Handler, * Michael Appel, \\ Robert Lew," Dale Greiner, ${ }^{*}$ John P. Mordes, ${ }^{*}$ and Aldo A. Rossini * \\ Departments of * Medicine, ${ }^{\S}$ Pathology, and "Biometry, University of Massachusetts Medical School, Worcester, Massachusetts 01655; \\ and ${ }^{\ddagger}$ Department of Microbiology and Immunology, University of South Carolina, Columbia, South Carolina 29208
}

\begin{abstract}
Dendritic cells (DC) present antigen and initiate $T$ cell-mediated immune responses. To investigate the possible association of autoimmunity with DC function, we compared the accessory activity of splenic DC from Wistar / Furth (WF) and diabetes-prone (DP) BioBreeding (BB) rats. The latter develop autoimmune diabetes and thyroiditis. DC function was quantified in vitro by measuring $T$ cell proliferation in mitogen-stimulated and mixed lymphocyte reactions. When purified without macrophage coculture, WF and DP DC displayed similar levels of accessory activity. In contrast, when purified by a method involving coculture with macrophages, DC from DP rats consistently displayed greater accessory activity. This finding could not be explained by morphological or phenotypic differences between DP and WF DC. In accessory activity assays performed after reciprocal DC cocultures with DP and WF macrophages, DP DC exhibited higher accessory activity irrespective of macrophage donor strain. We also compared the accessory activity of WF and DP DC cultured in the presence of conditioned medium and a mixture of IL-1 and GM-CSF. In all assays, DP DC exhibited higher accessory activity. In studies of $(W F \times D P) F_{1}$ hybrids, the high accessory activity of DP DC was observed to be heritable, and studies of WF and DP radiation chimeras indicated that the effect was an intrinsic property of the DP hematopoietic system. We conclude: $(a)$ splenic DC from DP and WF rats possess similar basal levels of accessory potency; (b) after interaction with macrophages, DC of DP origin are capable of greater stimulatory activity than are WF DC; and (c) the mechanism responsible for this phenomenon involves differential responsiveness of DP and WF DC to macrophage-derived factors such as IL-1 and GMCSF. (J. Clin. Invest. 1993. 91:2040-2048.) Key words: antigen presentation • autoimmunity $\bullet$ BB rat $\bullet$ dendritic cell • macrophage
\end{abstract}

\section{Introduction}

Dendritic cells (DC) ${ }^{1}$ are antigen-presenting cells (APC) specialized to initiate antigen-specific $T$ cell responses (1). T cell

Address reprint requests to Dr. Aldo A. Rossini, Diabetes Division, University of Massachusetts Medical School, II Biotech Park, 373 Plantation Street, Suite 218, Worcester, MA 01605.

Received for publication 27 July 1992 and in revised form 16 December 1992.

1. Abbreviations used in this paper: APC, antigen-presenting cell(s); BB, BioBreeding; BM, bone marrow; BPA, bovine plasma albumin;

J. Clin. Invest.

(C) The American Society for Clinical Investigation, Inc.

0021-9738/93/05/2040/09\$2.00

Volume 91, May 1993, 2040-2048 stimulation requires both antigen presentation to the $T$ cell receptor (TCR) in the context of MHC molecules and costimulatory signal(s), delivered by APC. In the absence of costimulatory signal $(\mathrm{s}) \mathrm{T}$ cells remain unresponsive $(2,3)$. In vitro, $\mathrm{DC}$, but neither B cells (4) nor macrophages (5), can induce primary $T$ cell responses in mitogenic systems $(5)$ and mixed leukocyte reactions (MLR) $(5,6)$. DC activity is highly sensitive to macrophage-derived cytokines ( 1 ). In vivo, DC capture, process, and present exogenous antigens to $T$ cells $(7,8)$. DC may also constitutively process and present self antigens (9). These characteristics suggest that DC could play a role in autoimmunity as presenters of self antigens.

We investigated DC from the diabetes-prone (DP) BioBreeding (BB) rat, an animal that develops both autoimmune diabetes and thyroiditis (10-12). Many studies have indicated that APC and T cells both play crucial roles in these autoimmune processes. The pathological substrate of BB rat diabetes is pancreatic islet inflammation (insulitis). Macrophages and DC are among the earliest infiltrating cellular constituents of insulitis (13-16). Neonatal thymectomy (17), selective T cell depletion (18), and silica treatment (19) in vivo all prevent diabetes. Concanavalin A (Con A)-activated T cells adoptively transfer both diabetes and thyroiditis to MHC-compatible hosts $(20,21)$. We undertook to determine if abnormalities of the APC system, specifically DC, exist in the BB rat.

Splenic DC were isolated from young nondiabetic DP-BB and normal histocompatible Wistar/Furth (WF) rats. The stimulatory capability of these DC was then measured in T cell proliferation assays. The results indicate that, subsequent to interaction with macrophages and/or macrophage-derived factors, DC from DP rats have intrinsically greater capability for accessory activity than do DC of WF origin.

\section{Methods}

\section{Animals}

Diabetes-prone (DP) and diabetes-resistant (DR) BB Worcester (Wor) rats $\left(R T 1^{\mathrm{u} / \mathrm{u}}\right)$ were obtained from a viral antibody-free colony at the University of Massachusetts, Worcester $(22,23)$. About $60-80 \%$ of both male and female DP rats become diabetic between 60 and $120 \mathrm{~d}$ of age; $<1 \%$ of DR rats become hyperglycemic. Inbred WF $\left(R T 1^{u / u}\right)$, Copenhagen (COP; RT1 $1^{\mathrm{a} / \mathrm{a}}$ ), M520 $\left(\mathrm{RT}^{\mathrm{b} / \mathrm{b}}\right)$, Lewis (LEW, RT1 ${ }^{1 / 1}$ ), and Fisher $344\left(R T 1^{1 / 1}\right)$ rats were obtained from the National Cancer Institute. $(\mathrm{WF} \times \mathrm{DP}) \mathrm{F}_{1}$ hybrid rats were produced in our colony using WF males.

Cell culture conditions

All cell cultures were carried out in media consisting of RPMI-1640 supplemented with $2 \mathrm{mM}$ L-glutamine, $100 \mu \mathrm{g} / \mathrm{ml}$ streptomycin, 100 $\mathrm{U} / \mathrm{ml}$ penicillin, and $10 \%$ heat-inactivated horse serum (Gibco Labora-

$\mathrm{DC}$, dendritic cell(s); DP and DR, diabetes prone and diabetes resistant; HD and LD, high density and low density; LNC, lymph node cell(s); MLR, mixed leukocyte reaction(s); PEC, peritoneal exudate cell(s); SPC, spleen cell(s); TCR, T cell receptor; WF, Wistar/Furth; Wor, Worcester. 
tories, Grand Island, NY). Incubation was at $37^{\circ} \mathrm{C}$ in a humidified atmosphere of $7 \% \mathrm{CO}_{2}$ in air.

\section{DC preparation}

Depending on the individual experiment, most DC were prepared using one or a combination of three procedures: FcR rosetting (5), silica pulsing (24), and adherence to plastic (5). These procedures differed principally with respect to the method and timing of macrophage depletion. The initial isolation of DC was the same in all cases (5). Spleens of 30-45-d-old rats were removed aseptically and the cells separated on a discontinuous density gradient $(\rho=1.086 \mathrm{~g} / \mathrm{ml})$ of bovine plasma albumin (BPA, Armour Pharmaceutical Co., Biochemical Division, Kankakee, IL) into two fractions. The high density spleen cell (HD-SPC) fraction contained mainly small T and B cells, erythrocytes, and debris; the low density (LD-SPC) fraction contained mainly macrophages, DC, and large T and B cells. The LD-SPC fraction then underwent further treatment to enrich for $D C$ by one of the three methods ( FcR rosetting, silica treatment, or adherence to plastic) described below. As part of all three methods, radiosensitive cells were eliminated by $\gamma$-irradiation $\left(1,000 \mathrm{R},{ }^{137} \mathrm{Cs}\right.$, Gammacell 40 , Atomic Energy of Canada) followed by $48-50 \mathrm{~h}$ of culture and a second BPA fractionation.

$F c R$ rosetting. This method of DC purification avoided culture of $D C$ in the presence of macrophages and was used to obtain DC for studies of basal accessory activity. Immediately after isolation, LD-SPC were depleted of macrophages by exploiting the high levels of FcR receptor expression on macrophages; FcR are low or absent on DC (5). Sheep red blood cells (SRBC) were incubated for $30 \mathrm{~min}$ at $4^{\circ} \mathrm{C}$ in the presence of subagglutinating doses of rabbit $\alpha$-SRBC antiserum. FcRSRBC ( $5 \% \mathrm{vol} / \mathrm{vol})$ were added to equal volumes of LD-SPC cells $(20$ $\times 10^{6}$ cells $/ \mathrm{ml}$ ), incubated for $20 \mathrm{~min}$ on ice to form rosettes linking macrophages to the immunoglobulin-coated SRBC. The rosetted and nonrosetted cell populations were gently resuspended and fractionated on a BPA density gradient as described above. SRBC-macrophage rosettes sedimented in the pellet. The FCR ${ }^{-}$LD fraction then underwent final irradiation, culture, and BPA gradient fractionation as described above.

Silica treatment. This method of DC purification included culture of DC in the presence of activated macrophages. After isolation, LDSPC were cultured for $36-38 \mathrm{~h}$ and then pulsed with silica $(1 \mathrm{mg} / \mathrm{ml}$, Sigma Chemical Co., St. Louis, MO) for an additional 10-12 h. This procedure exploits the fact that macrophage phagocytic activity persists after prolonged culture, whereas DC lose phagocytic capability after 10-12 hours (25). During the final BPA fractionation, silica-laden macrophages segregated into the HD fraction and were removed. DC isolated by this procedure were cocultured with macrophages for a total of 48-50 h. "Conditioned medium" used in certain experiments was obtained by collecting supernatants of silica-treated LD-SPC cell cultures (26). Cells were spun down and culture supernatants were frozen immediately, stored at $-70^{\circ} \mathrm{C}$, and used within $2 \mathrm{wk}$. Fresh culture medium ( $10 \% \mathrm{vol} / \mathrm{vol})$ was added to conditioned media just before use.

Purification of DC using adherence: syngeneic and reciprocal macrophage coculture experiments. The method was devised to compare WF and DP DC cultured in the presence of either syngeneic or heterologous macrophages. LD-SPC from DP and WF rats were cultured for 4-5 h and separated into two fractions by plastic adherence. The nonadherent fraction contained mainly DC and T and B blasts; the adherent fraction contained mainly macrophages. Nonadherent cells were recovered by swirling the dish and gently washing with warm medium $\left(37^{\circ} \mathrm{C}\right)$ four times. Syngeneic (DP-DP; WF-WF) and reciprocal (DPWF; WF-DP) DC-macrophage cocultures were then performed. After 30-36 $\mathrm{h}$ of incubation, both the syngeneic and reciprocal cocultures were pulsed with silica for 10-12 $\mathrm{h}$ and the DC purified as described above.

Viability and yield of $D C$. After purification by any of these methods, DC viability was always $>98 \%$ by trypan blue dye exclusion. Every preparation of DC was examined by light microscopy; the number of cells displaying typical DC morphology was determined and used to calculate the concentration of DC to be used in each experiment. Consistent with previous reports (27), DC yield was 0.02 to $0.1 \%$ of the initial total spleen cell number.

\section{Preparation of responder cells}

Cervical and mesenteric lymph nodes were removed aseptically from male rats. DC and macrophages were removed by fractionation on a BPA density gradient. The efficacy of this procedure in producing "accessory cell-depleted" responder populations has been documented previously $(5,27)$ and was confirmed in our experiments. High density lymph node cells (HD-LNC) were collected from the pellet, with an average recovery of $65-80 \%$. In some experiments, $T$ cell enrichment was performed by eliminating B cells with a biotin-avidin rosetting procedure using anti-rat Ig (28). Viability of purified T cells was $>95 \%$ by trypan blue dye exclusion. T cell purity was determined by

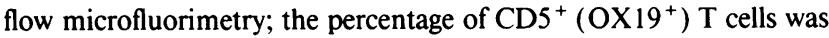
usually $92-96 \%$ and was never $<89 \%$.

\section{Preparation of peritoneal exudate cells (PEC)}

Resident PEC were recovered from exsanguinated animals by flushing the peritoneum with $20 \mathrm{ml}$ of cold Dulbecco's PBS. The cell suspension was enriched for macrophages by fractionation on a BPA density gradient. The LD-PEC preparations were examined by light microscopy and $\geq 95 \%$ of cells were found to exhibit typical macrophage morphology. This result was confirmed by nonspecific esterase staining and electron microscopy. LD-PEC were $>95 \%$ viable by trypan blue dye exclusion.

\section{Assays of DC accessory activity}

DC accessory activity was quantified by measuring the proliferative response of accessory cell-depleted HD-LNC or purified T cells cocultured in the presence of increasing concentrations of $\mathrm{DC}$. The following assay systems were used: $(a)$ oxidative mitogenesis using sodium periodate $\left(\mathrm{NaIO}_{4}\right) ;(b)$ lectin-stimulated mitogenesis using Con $\mathrm{A} ;(c)$ allogeneic and syngeneic MLR. These systems were selected on the basis of two previous observations $(5,27)$. First, accessory cell-depleted lymphocyte (HD-LNC or purified T cells) exhibit negligible proliferation in the absence of DC. Second, the magnitude of T cell proliferation varies as a function of the number of added DC.

Assay cultures were performed in triplicate in flat-bottomed 96-well plates (Falcon, Becton Dickinson Labware, Lincoln Park, NJ) in 0.2 $\mathrm{ml}$ of culture medium as previously described $(5,6,27)$. All assays were performed using $5 \times 10^{5}$ responder cells per well and graded doses of splenic DC. In each experiment, DC from both WF and DP rats were isolated and assayed in parallel. To quantify $T$ cell blastogenesis, cultures were pulsed during the final $4 \mathrm{~h}$ of incubation with medium $(5 \mu \mathrm{l}$ per well) containing $\left[{ }^{3} \mathrm{H}\right]$ thymidine ( TdR) and unlabeled TdR at saturating conditions ( $f$ inal TdR concentration in each well: $1 \times 10^{-5} \mathrm{M} ; \mathrm{sp}$ act $300 \mathrm{mCi} / \mathrm{mM})$. Under these conditions, $\left[{ }^{3} \mathrm{H}\right] \mathrm{TdR}$ incorporation correlates with the number of responding cells as determined by autoradiography (29). [ $\left.{ }^{3} \mathrm{H}\right] \mathrm{TdR}$ incorporation in the cultured cells was measured using a liquid scintillation counter. The incorporated radioactivity was calculated as the average number of counts per minute ( $\mathrm{cpm})$ of triplicate cultures.

Periodate assays. In the $\mathrm{NaIO}_{4}$ assays $(27,30)$, responder cells were incubated on ice at a concentration of $1 \times 10^{7}$ cells $/ \mathrm{ml}$ in freshly prepared PBS containing $1.2 \mathrm{mM} \mathrm{NaIO}_{4}$ (Fisher Scientific Co., Pittsburgh, PA). After incubation for $15 \mathrm{~min}$, periodate-treated cells were washed twice in RPMI-1640 and resuspended in culture medium in the presence or absence of DC. Cell proliferation was measured by incorporation of $\left[{ }^{3} \mathrm{H}\right] \mathrm{TdR}$ after $48-52 \mathrm{~h}$ of culture. In the absence of DC, the background $\left[{ }^{3} \mathrm{H}\right] \mathrm{TdR}$ incorporation of periodate treated responder cells was usually $<500 \mathrm{cpm}$ and $<10 \%$ of DC-stimulated responses.

Lectin-stimulated assays. In these assays, responder cells were incubated with Con A at a concentration of $5 \mu \mathrm{g} / \mathrm{ml}$ for 30-60 min at $37^{\circ} \mathrm{C}$. They were then added without washing to $\mathrm{DC}$, yielding a final Con A concentration of $2.5 \mu \mathrm{g} / \mathrm{ml}$. $\left[{ }^{3} \mathrm{H}\right] \mathrm{TdR}$ uptake was measured 
after 62-66 $\mathrm{h}$ of culture. In the absence of DC, the response of Con A-treated responders was usually $<500 \mathrm{cpm}$.

$M L R$. In MLR, responder cell proliferation was measured in both allogeneic and syngeneic systems. Incubation times were 72-76 and 96-98 $\mathrm{h}$ for allogeneic and syngeneic MLR, respectively. Background response was usually $<200 \mathrm{cpm}$.

Choice of standard conditions. For each assay system, the time of incubation was chosen on the basis of preliminary experiments using WF DC and responder cells. Incubation times and mitogen concentrations were selected to generate optimal dose response curves with graded concentrations of DC. Standard deviations associated with the average of the triplicates in each assay usually ranged between $10 \%$ and $15 \%$ of the mean.

\section{Flow microfluorimetry}

Cell suspensions ( $10^{5}$ to $10^{6}$ cells per sample) were incubated for 30-50 min at $4^{\circ} \mathrm{C}$ with the appropriate monoclonal antibody ( $\left.\mathrm{mAb}\right)$, washed twice with RPMI-1640 containing $1 \%$ fetal calf serum and $0.05 \%$ $\mathrm{NaN}_{3}$, and developed for fluorescence as previously described (31). Mouse anti-rat antibodies used included MRC OX1 (anti-leukocyte common antigen, LCA), MRC OX6 (anti-Ia), MRC OX19 (antiCD5), and R73.1 (anti- $\alpha / \beta$ T CR); all were purchased from Bioproducts for Science, Inc., Indianapolis, IN. The A3C (mouse IgG $_{2 \mathrm{a}}$ ) and $\mathrm{C} 11 \mathrm{~B}$ ( mouse $\mathrm{IgG}_{1}$ ) $\mathrm{mAb}$ have been developed in Dr. Bowers' laboratory; both antibodies are highly specific for rat DC. Neither binds to macrophages; A3C weakly stains B cells (unpublished observations). Mouse $\mathrm{IgG}_{1}$ and rat IgG were obtained from Sigma Chemical Co. Hybridomas producing the anti-RT7.1 (BC84) and anti-RT7.2 (8G6.1) rat monoclonal antibodies are maintained in our laboratory (32). Analyses were performed on FACScan (Becton Dickinson, Mountain View, CA). A minimum of 5,000 cells per sample were analyzed.

\section{Bone marrow chimeras}

In certain experiments, DC obtained from bone marrow (BM) chimeras were studied. BM donors were 30-45-d-old DP or WF rats that were depleted of $T$ cells by intraperitoneal injections of $0 \times 19$ pan-T $\mathrm{mAb} 72,48,24$, and $12 \mathrm{~h}$ before use. BM was obtained by flushing femurs and tibias with RPMI-1640. T cell depletion was confirmed by microfluorimetry using the R73.1 mAb on aliquots of donor cells. BM recipients were 28-30-d-old DP and WF rats that were fasted overnight, $\gamma$-irradiated (750 R), and then injected intravenously with 1.9-2.1 $\times 10^{8} \mathrm{BM}$ cells within $2-6 \mathrm{~h}$ of irradiation. Both reciprocal (WF $\rightarrow$ $\mathrm{DP}, \mathrm{DP} \rightarrow \mathrm{WF}$ ) and control syngeneic (WF $\rightarrow \mathrm{WF}$ and DP $\rightarrow$ DP) reconstitutions were performed. Experimental animals were analyzed four weeks after irradiation and BM reconstitution. The extent of chimerism was assessed by measuring the percentage of cells expressing the RT7.1 and RT7.2 rat alloantigens that are expressed by DP and WF rats, respectively (32). Both unfractionated spleen cells and splenic DC obtained from BM recipients were assayed.

\section{Statistics}

Analysis of variance was performed on each data set (33). The outcome analyzed was responder cell proliferation $\left(\left[{ }^{3} \mathrm{H}\right] \mathrm{TdR}\right)$ obtained by subtracting background $\mathrm{cpm}$ from test $\mathrm{cpm}$. The factors or main effects were: $(a) \mathrm{DC}$ donor strain, $(b) \mathrm{DC} /$ responder cell ratio, and $(c)$ day-to-day variability in $\mathrm{cpm}$ associated with individual experiments. The analysis of variance accounted for all three factors and their interactions simultaneously, with the primary goal of finding statistically significant main effects. In simpler terms, a statistically significant difference between DP and WF DC responses means that, as a group, the response of one strain averaged over all DC/responder ratios (excluding the zero point where no responders were present) was different. Parametric data are presented as means \pm SEM.

\section{Results}

The basal levels of accessory activity of DP and WF dendritic cells are similar. To determine basal levels of WF and DP DC accessory activity, DC were isolated using the FcR rosetting procedure that did not involve coculture of $\mathrm{DC}$ with macrophages. This procedure was chosen because DC are known to be extremely sensitive to the stimulatory effects of macrophage-derived factors (1). DC accessory activity was measured as $T$ cell proliferation in an allogeneic MLR. We found that the basal levels of accessory activity of DC from DP and WF rats were comparable (Fig. $1 A$ ).

After coculture in the presence of macrophages, splenic DP $D C$ exhibit greater stimulatory activity than do WF DC. We next tested the accessory activity of DC that had been cultured with macrophages pulsed with silica. Assayed in an MLR, macrophage-cocultured DC of DP origin exhibited greater capacity than WF DC to stimulate responder cells from COP donors (Fig. $1 B$ ).

This result was subsequently confirmed and expanded in several assays. These included MLR using purified T cells from MHC-incompatible M520, LEW, and COP rats (Fig. 2, $A, B$,

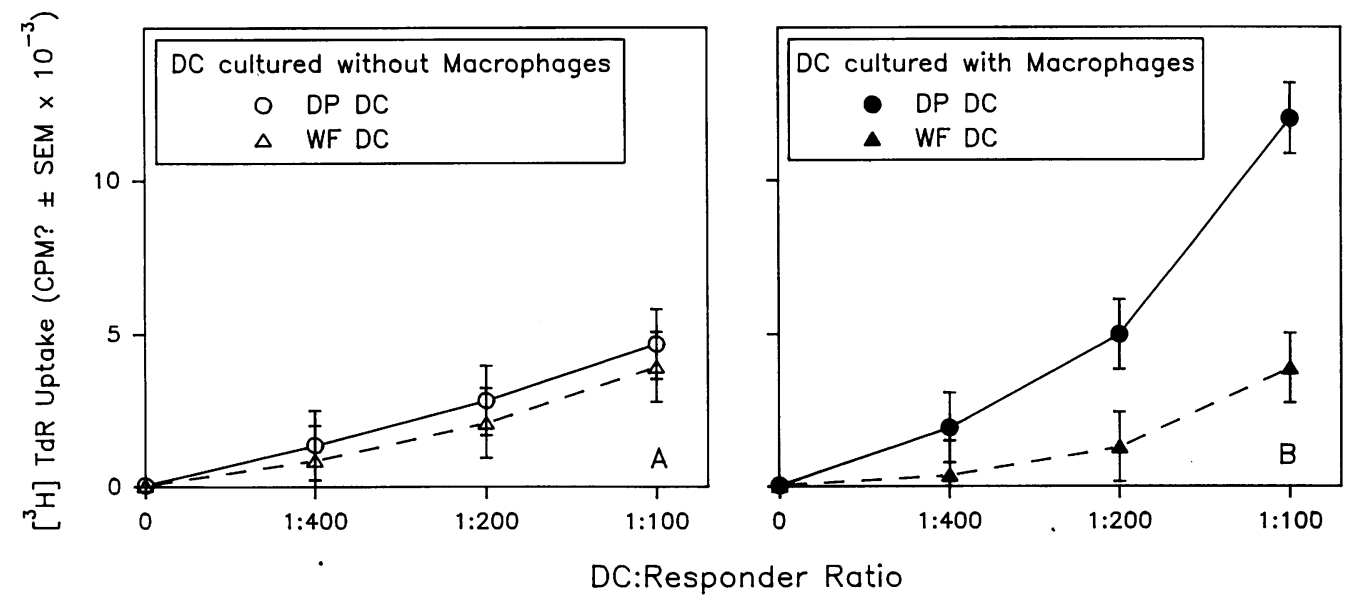

Figure 1. Basal DC stimulatory activity. DC were isolated in parallel by two different purification procedures as described in Methods. The first eliminated macrophages before LD-SPC culture (FcR rosetting, panel $A$ ). The second involved DC coculture with activated macrophages (silica treatment, panel $B$ ). The stimulatory activity of DC isolated by these two different methods was assessed in parallel by measuring responder cell proliferation $\left(\left[{ }^{3} \mathrm{H}\right] \mathrm{TdR}\right.$ uptake) in an allogeneic

MLR, using COP HD-LNC as responders $\left(5 \times 10^{5}\right.$ per well). DP DC $(0)$ and WF DC $(\Delta)$ purified by the FcR rosetting method exhibited low levels of stimulatory activity that were statistically similar $(A)$. When isolated by the silica method, DC of DP origin $(\bullet)$ were more active than WF DC $(\triangle)$ isolated by the same method $(B, P<0.001)$. DP DC isolated by the silica pulse method were also more active than both DP and WF DC isolated by FcR rosetting $(P<0.001)$. Each data point represents the mean of four separate experiments performed as triplicate cultures. 
and $C$ ). Additional data indicative of high DP DC activity were also obtained in a syngeneic MLR using accessory cell-depleted HD-LNC from $(W F \times D P) F_{1}$ hybrid rats (Fig. $2 D$ ).

The results obtained in MLR were further confirmed in experiments using mitogenic systems. Oxidative treatment with $\mathrm{NaIO}_{4}$ specifically induces $\mathrm{T}$ cells to undergo a single blastogenesis cycle in the presence of DC (30). $\mathrm{NaIO}_{4}$-treated lymph node $\mathrm{T}$ cells from WF rats exhibited significantly higher levels of proliferation in response to DP DC than they did to WF DC (Fig. $3 A$ ). Finally, in the Con A-stimulated assay with WF $T$ cells as responders, the highest level of accessory activity was again associated with DC of DP origin (Fig. $3 \mathrm{~B}$ ).

$D C$ preparations from $W F$ and DP rats are similar in morphology, phenotype, and purity. To assess whether morphologic or phenotypic differences between DP and WF DC could account for their different levels of stimulatory activity, we analyzed silica-pulsed DC preparations by microscopy and flow microfluorimetry. DC preparations from DP and WF rats were morphologically similar by light microscopy. Electron microscopy of DC preparations from both strains revealed characteristic morphologic features including numerous projections, irregularly shaped nuclei, and scarce endoplasmic reticulum.

Surface phenotyping ( Table I) revealed that $>80 \%$ of both WF and DP DC isolated by silica pulse expressed MHC class II and ICAM-1; they also stained with both the A3C and C11B anti-DC antibodies. $T$ cell contamination, as assessed by antiTCR staining with the R73.1 mAb, was $<1 \%$ in both cases. These results indicate $>80 \%$ DC purity and phenotypic similarity of DP and WF DC preparations. Expression of MHC class II and ICAM-1, taking into account both fluorescence intensity and cell size, was therefore comparable on activated DP and
WF DC. This observation is consistent with the results of Koide et al. (34), who found comparable levels of Ia expression on both resting and IL-1 stimulated mouse DC.

Since macrophages reportedly inhibit DC stimulated T cell proliferation $(5,35)$, we also assessed our DC preparations for macrophage contamination. Cytospins $\left(3-5 \times 10^{4}\right.$ cells per slide) of DC and peritoneal macrophages (LD-PEC) from DP and WF rats were compared. DP and WF DC preparations isolated by all three methods contained $<6 \%$ macrophages by nonspecific esterase staining. Peritoneal macrophages, stained in parallel as a positive control, exhibited $>95 \%$ purity. The high purity and comparably low levels of contaminating macrophages in DP and WF DC preparations suggest that the number of contaminating macrophages is unlikely to account for the differential DC accessory activity we observed.

Macrophage-cocultured $D C$ from $D P-B B$ rats exhibit greater accessory activity than do WF DC irrespective of the strain of the macrophage donor. To determine whether our observations reflected a property of DC rather than a property of the macrophages with which they were cocultured, we developed a special cell culture system. Macrophages were removed from LD-SPC by plastic adherence; the nonadherent cell fraction containing DC was recovered. The percentages of adherent cells (macrophages) in LD-SPC preparations were comparable: $45 \pm 13 \%(n=6)$ in the case of the DP and $37 \pm 14 \%(n$ $=6$ ) in the case of the WF. The DP and WF DC isolated by this procedure exhibited basal levels of stimulatory activity that were comparable. The magnitude of this activity was similar to that exhibited by resting DC isolated by FcR rosetting and not exposed to macrophages in culture (see Fig. $3 \mathrm{~B}$ ).

Having established the equivalence of the initial isolation

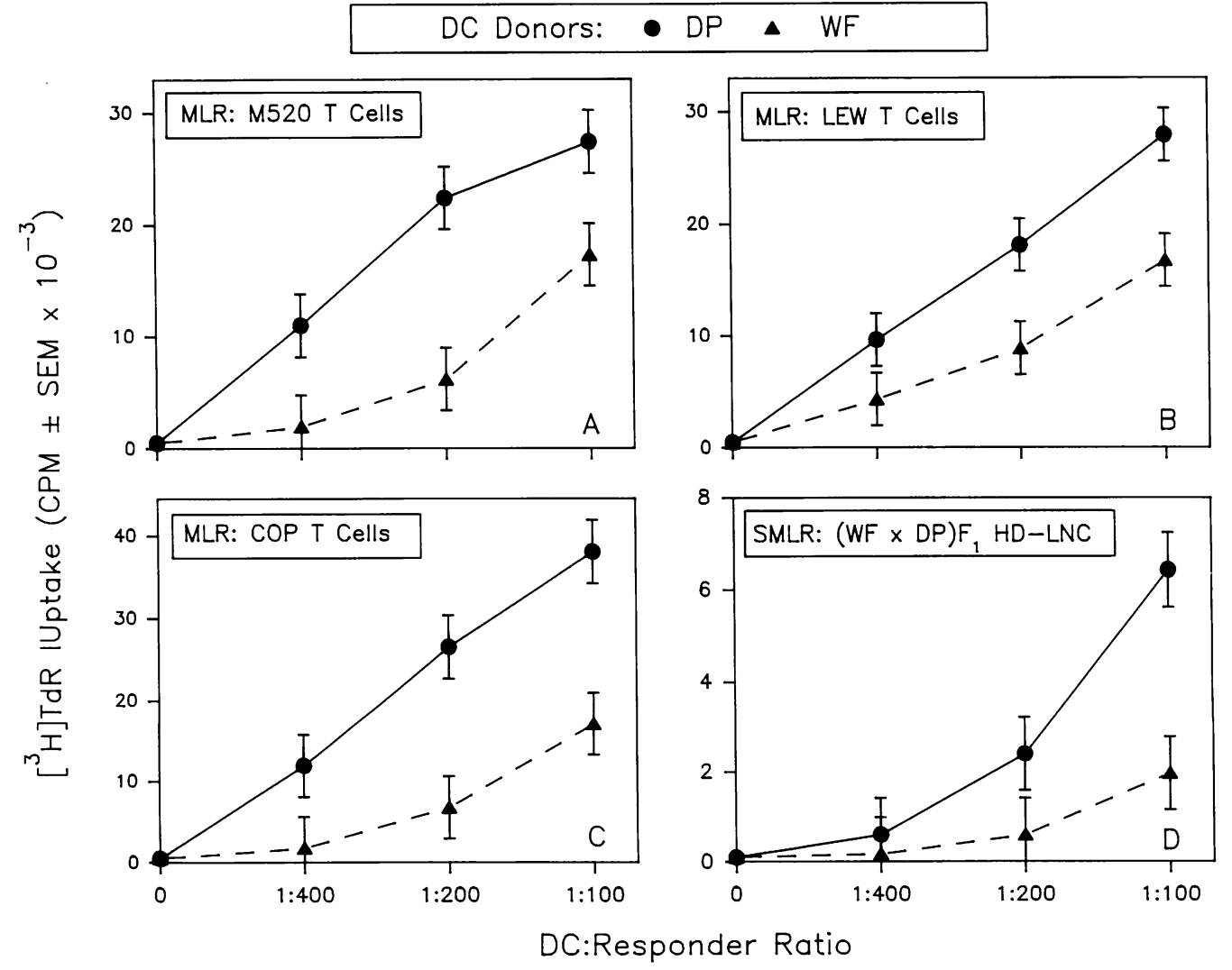

Figure 2. Differential stimulatory activity of DC from DP and WF rats in MLR. Splenic DC were isolated from DP and WF rats by macrophage-DC coculture and silica treatment as described in Methods. DC stimulatory activity was quantified as $\mathrm{T}$ cell proliferation ( $\left[{ }^{3} \mathrm{H}\right] \mathrm{TdR}$ uptake) in allogeneic $(A, B, C)$ or syngeneic $(D)$ systems. In the absence of $D C$, responder cells $\left(5 \times 10^{5}\right.$ per well $)$ exhibited negligible proliferation. $T$ cell proliferation varied as a function of DC number. DC of DP origin (•) consistently exhibited statistically significantly greater stimulatory activity than DC of WF origin $(\Delta)$. ( $A) \mathrm{Re}$ sponse of $\mathrm{M} 520\left(\mathrm{RT}^{\mathrm{b} / \mathrm{b}}\right) \mathrm{T}$ cells (DP DC vs. WF DC, $P$ $<0.001, n=4)$. (B) Response of LEW $\left(\mathrm{RT} 1^{1 / 1}\right) \mathrm{T}$ cells (DP vs. WF, $P<0.001$, $n=5$ ). (C) Response of COP T cells (DP vs. WF, $P$ $<0.001, n=4)$. (D) Re-

sponse of syngeneic (WF $\times$ DP) $F_{1}$ HD-LNC (DP vs. WF, $\left.P<0.02, n=4\right)$. Each data point represent the mean of separate experiments performed as triplicate cultures. 


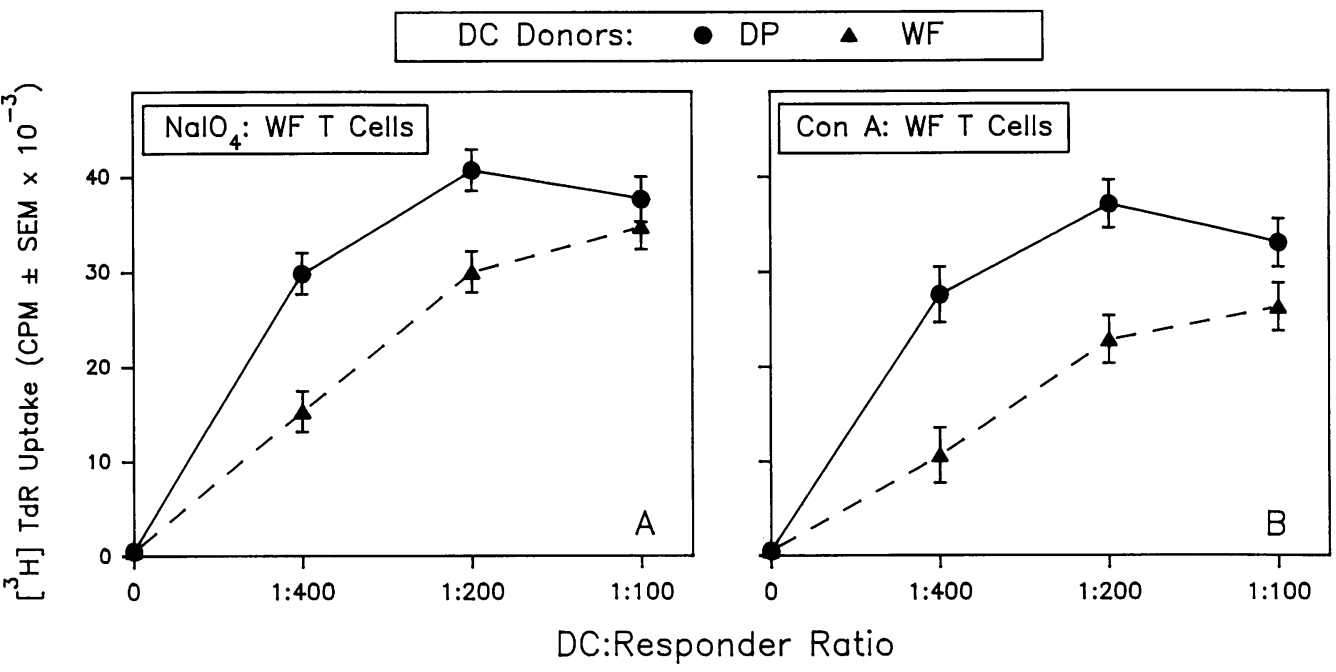

Figure 3. Differential stimulatory activity of DC from DP and WF rats in mitogenic assays. Splenic DC were isolated from DP and WF rats by macrophage-DC coculture and silica treatment. DC stimulatory activity was quantified as $\mathrm{T}$ cell proliferation $\left(\left[{ }^{3} \mathrm{H}\right] \mathrm{TdR}\right.$ uptake $)$ in response to $\mathrm{NaIO}_{4}(A)$ or Con $\mathrm{A}(B)$. The number of responder cells was constant $\left(5 \times 10^{5}\right.$ per well $)$. In the absence of DC, there was negligible $\mathrm{T}$ cell proliferation DC of DP origin $(\bullet)$ consistently exhibited significantly greater stimulatory activity than DC of WF origin ( $\Delta$ ).

( $A$ ) WF T cell response (DP vs. WF, $P<0.001, n=6$ ). ( $B$ ) Response of WF T cells (DP vs. WF, $P<0.001, n=4$ ). In each panel, data points represent the mean of separate experiments performed as triplicate cultures.

conditions, DP and WF DC depleted of adherent macrophages were then cocultured with syngeneic or heterologous macrophages using our standard conditions, pulsed with silica, and assayed for accessory activity. Again, DP DC exhibited greater $T$ cell stimulatory activity than WF DC irrespective of whether cocultures were performed with macrophages of DP or WF origin (Fig. 4).

$D C$ from $B B$ rats exhibit greater accessory activity than $W F$ $D C$ in the presence of either DP or WF LD-SPC culture supernatant. We next assessed the possibility that WF and DP macrophages differed with respect to the elaboration of inhibitory or stimulatory factors relevant to DC accessory activity. To do so, we recovered supernatants from WF or DP silica-treated LDSPC cultures (see Methods) and cultured macrophage-depleted LD-SPC from DP and WF rats in these "conditioned" media. Again, DC of DP origin exhibited greater stimulatory activity, regardless of the source of the conditioned medium in which they were cultured (Fig. 5, $C$ and $D$ ).

$D C$ from $B B$ rats exhibit greater accessory activity than $W F$ $D C$ in the presence of exogenous $I L-1$ plus GM-CSF. Since the data obtained to this point suggested that DP DC might be more sensitive to macrophage-derived factors, we tested the possibility directly by adding a mixture of human recombinant IL-1 $\beta(10 \mathrm{U} / \mathrm{ml})$ and mouse recombinant GM-CSF (25 U/ $\mathrm{ml}$ ) (Biosource Int., Camarillo, CA) to macrophage-depleted WF and DP LD-SPC. Each of these cytokines was used at concentrations previously shown to induce maximal DC stimulation (26). WF DC activity was stimulated two- to threefold, but again DC of DP origin exhibited much greater stimulatory activity despite the addition of equal amounts of IL-1 and GMCSF (Fig. $5 B$ ).

The high accessory activity of macrophage-cocultured DP $D C$ is a heritable characteristic. To investigate the genetic basis of DC stimulatory activity, we isolated splenic DC from (WF $\times$ DP) $F_{1}$ hybrids using the silica pulse method. $(W F \times D P) F_{1}$ hybrids do not become spontaneously diabetic (36). We tested DC from these hybrid rats in both the periodate assay using syngeneic responder cells (Fig. $6 A$ ) and in an MLR using COP-HD-LNC as responders (Fig. $6 \mathrm{~B}$ ). In both assays, the accessory activity observed using $(\mathrm{WF} \times \mathrm{DP}) \mathrm{F}_{1}$ hybrid $\mathrm{DC}$ was intermediate between the higher DP and lower WF levels. Interestingly, we also observed in the course of these same experiments that the accessory activity of $D C$ from the $D R B B$ rat was also intermediate between the DP and WF (Fig. 6).

Table I. Cell Surface Phenotype of Splenic DC and HD Spleen Cells

\begin{tabular}{llllrrrr}
\hline & & OX-1 & OX-6 & $\alpha$-ICAM-1 & A3C & C11B \\
\hline & & & & & $\%$ & & \\
RC & & & & & \\
& & & & & & \\
& DP-BB & $90 \pm 1(5)$ & $87 \pm 2(6)$ & $79 \pm 7(7)$ & $81 \pm 4(5)$ & $84 \pm 2(5)$ & $0 \pm 0(3)$ \\
HD spleen cells & WF & $88 \pm 3(5)$ & $83 \pm 6(6)$ & $72 \pm 6(7)$ & $86 \pm 4(4)$ & $85 \pm 3(4)$ & $1 \pm 1(3)$ \\
& DP-BB & $81 \pm 4(5)$ & $38 \pm 6(4)$ & - & $2 \pm 3(5)$ & $1 \pm 2(5)$ & $5 \pm 4(4)$ \\
& WF & $87 \pm 3(5)$ & $30 \pm 4(5)$ & - & $11 \pm 7(4)$ & $4 \pm 4(5)$ & $36 \pm 9(3)$
\end{tabular}

Phenotypic characterization of DC and HD-SPC from DP and WF rats. LD-SPC and HD-SPC were prepared by BPA density gradient centrifugation; DC were purified from the LD-SPC fraction by the silica procedure (See Methods). DC purity is indicated by staining with A3C and C11B, mAbs that are highly specific for DC (Dr. Bowers, unpublished observations). High levels of expression of both MHC class II (OX-6) and ICAM-1 are characteristic of DC. When DC were purified using FCR rosetting or a combination of adherence and silica pulsing, comparable levels of expression of both MHC class II and ICAM-1 were observed. The staining profile of HD-SPC from DP and WF are given for comparison. The low level of T lymphocytes (R73.1) in DP preparations reflects the T cell lymphopenia of these animals (10). 


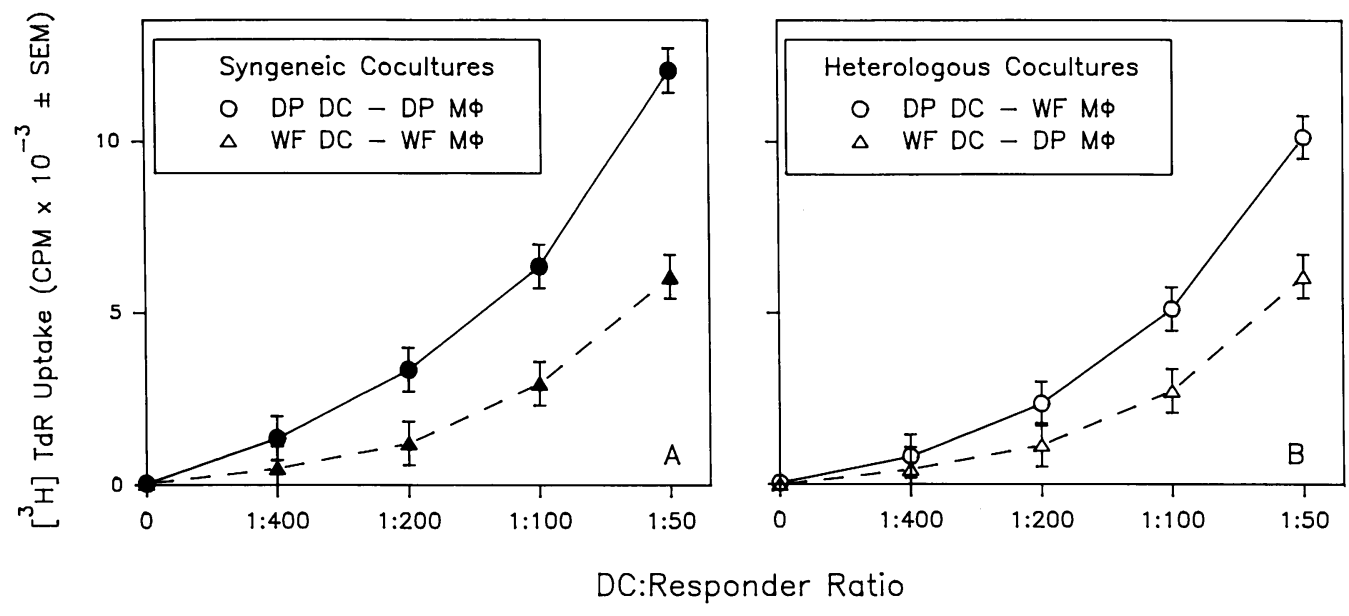

Figure 4. Stimulatory activity of DP and WF DC cocultured with either syngeneic or heterologous macrophages. LD-SPC were depleted of macrophages ( $M \Phi)$ by plastic adherence as described in the methods. Nonadherent cells were removed and LD-SPC cocultures then reestablished either syngeneically (DP DC + DP M $\Phi$ and WF DC + WF M $\Phi)$ or heterologously (DP DC + WF $M \Phi$, and WF DC + DP M $\Phi$ ). After coculture, DC were purified by silica pulse and their stimulatory activity quanti-

fied as responder cell proliferation $\left(\left[{ }^{3} \mathrm{H}\right] \mathrm{TdR}\right.$ uptake $)$ in an allogeneic MLR, using Fisher $344 \mathrm{HD}-\mathrm{LNC}$ as responders $\left(5 \times 10^{5}\right.$ per well $) .(A)$ Syngeneic cocultures. DC from DP $(\bullet)$ exhibited significantly greater stimulatory activity than DC of WF $(\Delta)$ origin (DP DC vs. WF DC; $P$ $<0.001)$. (B) Heterologous cocultures. DP DC $(O)$ were again more active than DC of WF $(\triangle)$ origin (DP vs. WF, $P<0.001$ ). Each data point represents the mean of four separate experiments performed as triplicate cultures.

The high accessory activity of macrophage-cocultured DP $D C$ is intrinsic to the DP hemopoietic system. To investigate whether the high accessory activity we observed using DP DC is an inherent characteristic of the hematopoietic system, DP and WF BM chimeras were generated as described in the Methods. DC precursors reside in the $\mathrm{BM}(1,37,38)$, and the turnover interval for splenic DC is $\sim 2-4$ wk (39). Our experimental animals were studied $4 \mathrm{wk}$ after irradiation and BM reconstitution. This helped assure both that the chimeras' DC would be of donor origin and that they had matured in the radioresistant environment of the host. Chimerism of both total spleen cells and purified DC was confirmed by measuring the percentage of cells expressing the RT7.1 and RT7.2 rat alloantigens that are expressed by DP and WF rat DC, respectively (32). In three separate MLR assays and at two DC/responder cell ratios, we observed that DC of DP origin exhibited higher accessory activity than DC of WF origin, irrespective of the host in which they had matured (Fig. 7).

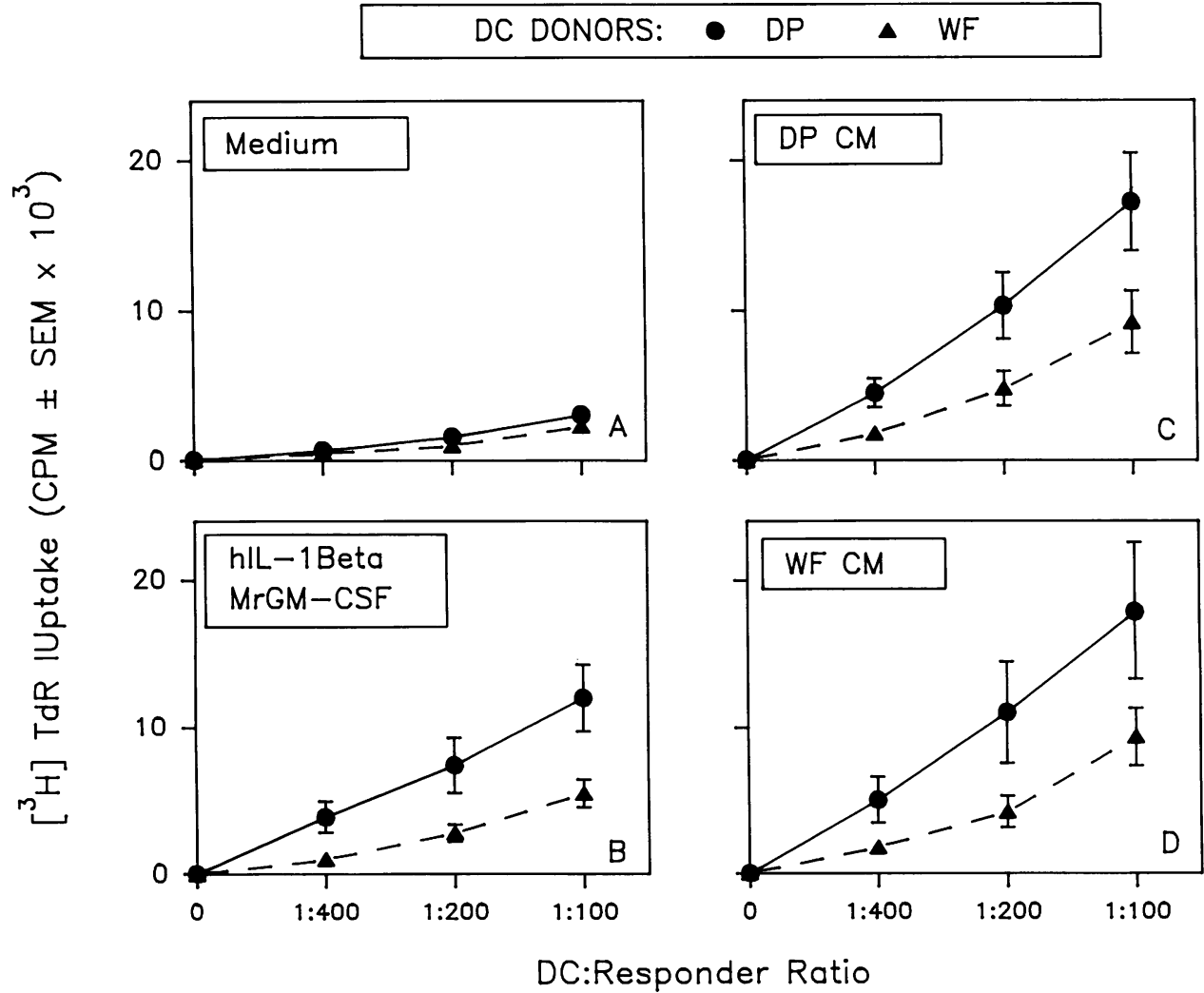

Figure 5. Differential effect of macrophage-derived cytokines on stimulatory activity of DP and WF DC. LD-SPC depleted of macrophages by FcR rosetting were incubated for $36 \mathrm{~h}$ in the presence of various added reagents: $(A)$ Unmodified culture medium. $(B)$ a mixture of human recombinant IL-1 $\beta$ ( $10 \mathrm{U} /$ $\mathrm{ml}$ ) and mouse recombinant GMCSF $(25 \mathrm{U} / \mathrm{ml})$. (C) Conditioned culture medium (see Methods) from silica-treated LD-SPC from DP rats. (D) Conditioned culture medium from WF rats. DC stimulatory activity was quantified as responder cell proliferation $\left(\left[{ }^{3} \mathrm{H}\right] \mathrm{TdR}\right.$ uptake $)$ in an allogeneic MLR, using Fisher 344 HD-LNC responders $\left(5 \times 10^{5}\right.$ per well). DP DC $(\bullet)$ consistently exhibited greater stimulatory activity than DC of WF ( $\triangle$ ) origin (DP vs. WF, $P<0.001$ for $B-D, P=\mathrm{NS}$ for $A$ ). Each data point represents the mean of three separate experiments performed as triplicate cultures. 


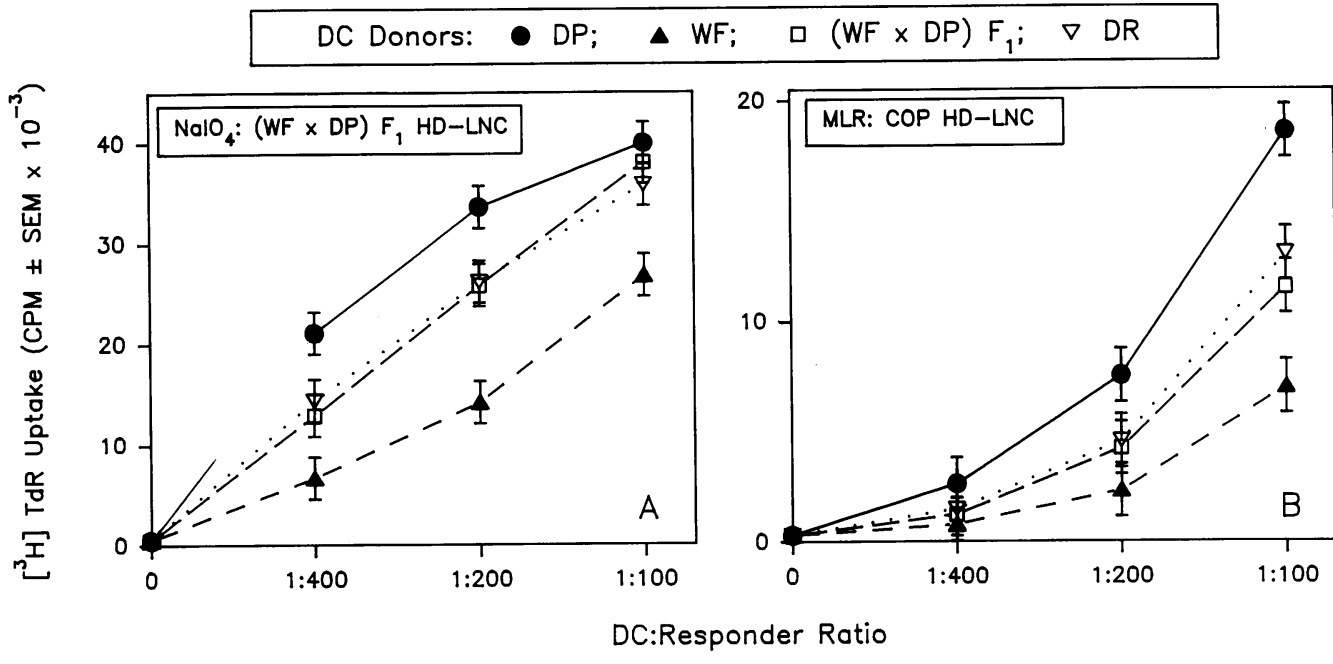

Figure 6. Stimulatory activity of DC from $(\mathrm{DP} \times \mathrm{WF}) \mathrm{F}_{1}$ hybrids and from $D R$ rats. DC were purified using macrophage coculture and silica pulse as described in the Methods. DC stimulatory quantified as HD-LNC proliferation $\left(\left[{ }^{3} \mathrm{H}\right] \mathrm{TdR}\right.$ uptake $)$ in a $\mathrm{NaIO}_{4}$ assay $(A)$ or an allogeneic $\operatorname{MLR}(B)$. DC of DP origin $(\bullet)$ consistently exhibited significantly greater stimulatory activity than DC of WF origin ( $\triangle$ ); DC from $(\mathrm{WF} \times \mathrm{DP}) \mathrm{F}_{1}$ hybrids ( $\square$ ) possessed intermediate levels of activity, differing significantly from both DP and WF DC. The stimulatory activity of $\mathrm{DC}$ from $\mathrm{DR}$ rats $(\nabla)$ was similar to that of $(\mathrm{WF} \times \mathrm{DP}) \mathrm{F}_{1}$ hybrids. $(A)(\mathrm{WF} \times \mathrm{DP}) \mathrm{F}_{1}$ HD-LNC responders $\left(\mathrm{DP}\right.$ vs. WF, $P<0.001 ; \mathrm{DP}$ vs. $\mathrm{F}_{1}$ and DR, $P<0.01$; WF vs. $\mathrm{F}_{1}$ and DR, $P<0.001$; DP vs. $\left.\mathrm{F}_{1}, P=\mathrm{NS}\right)(B) \mathrm{COP}$ HD-LNC responders (DP vs. WF, $P<0.001 ; \mathrm{DP}$ vs. $\mathrm{F}_{1}$ and $\mathrm{DR}, P<0.001$; WF vs. $\mathrm{F}_{1}$ and $\mathrm{DR}, P<0.01$; DR vs. $\left.\mathrm{F}_{1}, P=\mathrm{NS}\right)$. Each point represents the mean of separate experiments performed as triplicate cultures.

\section{Discussion}

These studies investigated the hypothesis that susceptibility to autoimmunity in DP BB rats is associated with abnormalities of DC function. Our results demonstrate that, after culture in the presence of macrophages or their secretory products, DC from DP rats exhibit greater in vitro stimulatory activity than DC from normal, histocompatible WF rats.
Using DC isolated without macrophage coculture, we found that the basal accessory activity of DP and WF DC were comparable. We interpret this result to indicate that DP and WF DC behave similarly with respect to basal stimulatory activity. In contrast, after coculture with activated macrophages, splenic DC from DP rats consistently displayed greater in vitro stimulatory activity than DC from WF rats. This characteristic was evident in both mitogenic and MLR systems.
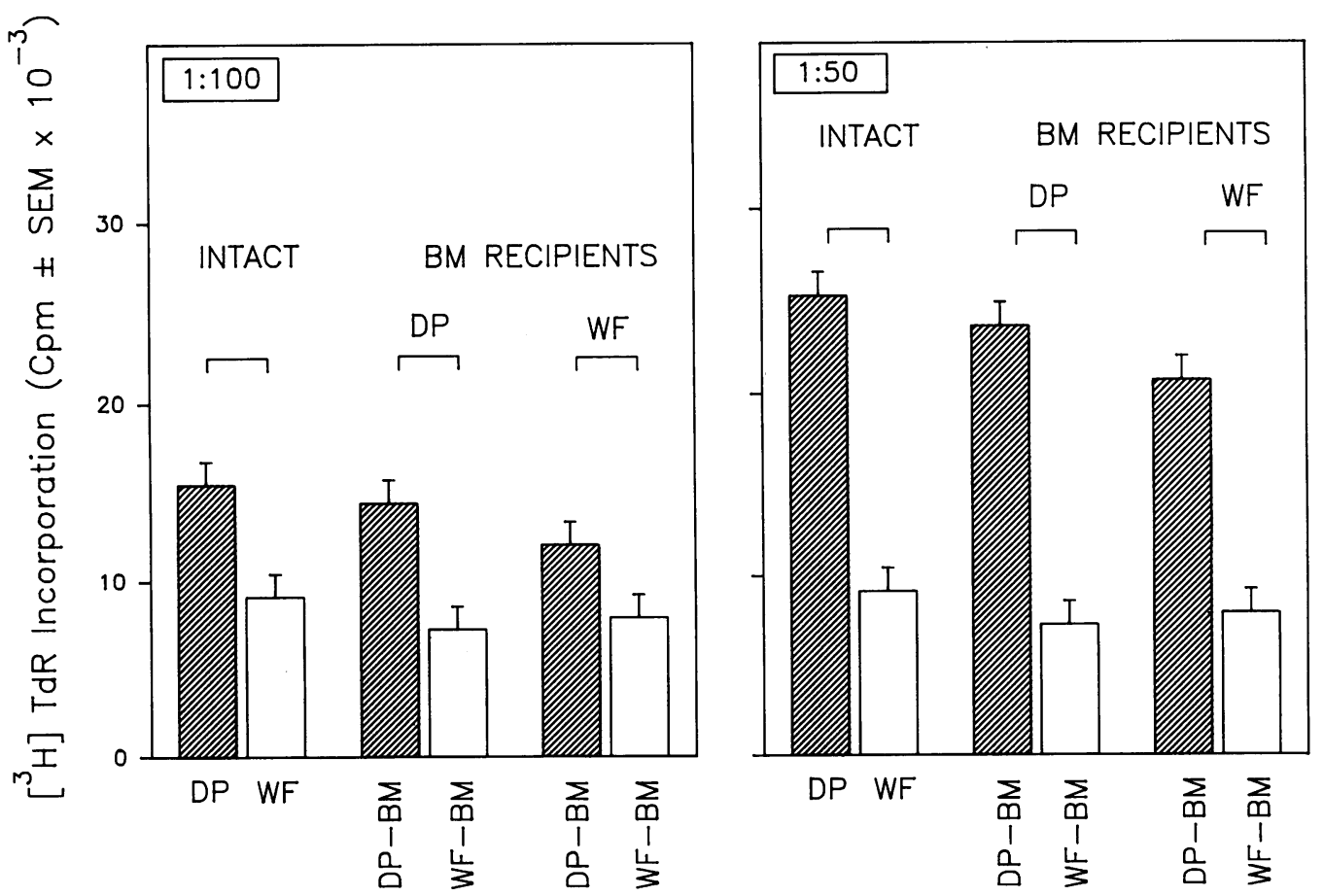

Figure 7. Stimulatory activity of DC from DP and WF reciprocal bone marrow chimeras. Splenic DC were obtained by the silica-pulse method from intact, unirradiated DP and WF rats and from irradiated, bone marrow reconstituted syngeneic (DP $\rightarrow$ DP and WF $\rightarrow$ WF) and reciprocal (DP $\rightarrow$ WF and WF $\rightarrow$ DP) chimeras. DC stimulatory activity was quantified as responder cell proliferation in an allogeneic MLR, using COP HD-LNC responders ( 5 $\times 10^{5}$ per well). Left and right panels present data obtained at DC/responder ratios of 1:100 and 1:50, respectively. DC of DP origin are indicated by filled bars and DC of WF origin by open bars. DC donors are indicated above the bars. DC of DP origin consistently exhibited statistically significantly greater stimulatory activity than DC of WF origin, irrespective of the environment in which they had matured. (Intact DC donors: DP vs. WF, $P<0.0001$ at both DC/responder ratios; recipients of DP-BM: $P<0.0001$; recipients of WF BM: $P<0.0002$ ). Each bar represents the mean of three separate experiments performed as triplicate cultures. 
Additional experiments established that this differential activity persisted when DP and WF DC were $(a)$ reciprocally cocultured with DP or WF macrophages and $(b)$ incubated with conditioned medium derived from either DP or WF LDSPC. These results indicate that properties inherent to DP vs. WF macrophages are unlikely to account for the difference between DP and WF DC accessory capability. This interpretation is strengthened further by the results of our assays performed using a mixture of IL-1 and GM-CSF, cytokines previously shown to account for most of the stimulatory activity of silica-treated LD-SPC culture supernatants (26). When DC from DP and WF rats were treated with a mixture of IL-1 and GM-CSF at doses corresponding to maximal stimulation, DP again exhibited greater stimulatory activity than WF DC. These findings suggest that DC from DP-BB rats are inherently more responsive to macrophage-derived factors than WF DC.

Our data do not exclude differential cytokine production by DP and WF macrophages. Stimulated DP-BB macrophages reportedly produce increased amounts of TNF (40). DP-BB macrophages also appear to inhibit mitogen-stimulated $\mathrm{T}$ cell responses by an undefined mechanism $(41,42)$. Given these observations, one might speculate that the DC-macrophage axis in the DP rat harbors abnormalities in both of its components. It is important to stress, however, that macrophage abnormalities alone could not account for the differential activity of DP and WF DC in vitro that we have observed.

Studies of $(W F \times D P) F_{1}$ hybrids, DR BB rats, and DP-WF reciprocal $B M$ chimeras indicate that the differential activity of DP and WF DC is genetically determined. The cellular basis of these findings appears to be intrinsic to the hemopoietic system and is not influenced by the radioresistant maturational milieu.

Possible involvement of DC in the development of autoimmune processes has been suggested previously. Voorbij et al. (16) and Voorby et al. (43) have shown in DP-BB rats that DC are the first cells to infiltrate both islets and thyroid, suggesting their involvement in the initiation phase of both diseases. Georgiou and Bellgrau (44) have documented a defect in APC function in the BB rat thymus and suggest that such a defect might be more widespread. Finally, diabetes may be prevented in DP rats by in vivo treatment with silica, probably by interfering with antigen processing and presentation (19). In the nonobese diabetic mouse, another animal model of autoimmune diabetes, a preliminary report by Clare-Salzer et al. (45) suggests a possible role of DC in tolerization. Footpad injection of DC from peripancreatic lymph nodes of nonobese diabetic mice with active insulitis reportedly conferred resistance to spontaneous and adoptively transferred diabetes. Interestingly, DC preparations derived from other lymphoid sites were not effective.

Our finding that DC from DP-BB rats become relatively hyperactive in response to macrophage-derived factors is compatible with two major hypotheses invoked to explain cell mediated autoimmune phenomena. Hyperactive DC could render immunogenic the constitutive tolerogenic presentation of self to the immune system. Alternatively, hyperactive DC could present tissue-specific neoantigens postnatally expressed or resulting from specific or nonspecific destruction, e.g., viral infection of $\beta$ cells. One may further speculate that the comparatively modest response of WF DC in response to cytokines could in part explain WF refractoriness to insulin-dependent diabetes mellitus and other autoimmune disorders. Evaluation of these possibilities will require extensive additional investigation and, in particular, expansion of the scope of study to include additional rat strains.

Interestingly, it has been recently shown that human thyroid epithelial cells produce IL-1 in the course of autoimmune thyroiditis (46). Furthermore, a human pancreatic beta cell line reportedly secretes IL-1 in response to viral infection (47). These observations suggest that both tissues could directly stimulate resident DC in the absence of other immune cell interactions. This could also account for the vulnerability of these tissues as targets of autoimmunity. The possibility that a defect in DC activity may underlie or contribute to susceptibility to autoimmune diabetes and thyroiditis warrants further study.

\section{Acknowledgments}

We thank Linda Leehy, Linda Paquin, and Olita Treimanis for technical assistance; Marcia McFadden and Tammy Kunst for help with the microfluorimetric analysis; and Dr. E. A. Goodell and Janet Stoltenborg for assistance in developing DC isolation method.

This study was supported in part by grants DK36024, DK41235, DK30657, and DK36042 from the National Institutes of Health, and by a grant from the Juvenile Diabetes Foundation. Dr. A. Tafuri is recipient of a Juvenile Diabetes Foundation postdoctoral research fellowship.

\section{References}

1. Steinman, R. M. 1991. The dendritic cell system and its role in immunogenicity. Annu. Rev. Immunol. 9:271-296.

2. Geppert, T. D., L. S. Davis, H. Gur, M. C. Wacholtz, and P. E. Lipsky. 1990. Accessory cell signals involved in T-cell activation. Immunol. Rev. 117:566.

3. Schwartz, R. H. 1990. A cell culture model for T lymphocyte clonal anergy. Science (Wash. DC). 248:1349-1356.

4. Metlay, J. P., E. Puré, and R. M. Steinman. 1989. Control of the immune response at the level of antigen-presenting cells: a comparison of the function of dendritic cells and B lymphocytes. Adv. Immunol. 47:45-116.

5. Klinkert, W. E. F., J. H. LaBadie, and W. E. Bowers. 1982. Accessory and stimulating properties of dendritic cells and macrophages isolated from various rat tissues. J. Exp. Med. 156:1-19.

6. Green, J., and R. Jotte. 1985. Interactions between T helper cells and dendritic cells during the rat mixed lymphocyte reaction. J. Exp. Med. 162:15461560 .

7. Liu, L. M., and G. G. MacPherson. 1991. Lymph-borne (veiled) dendritic cells can acquire and present intestinally administered antigens. Immunology. 73:281-286.

8. Crowley, M. T., K. Inaba, and R. M. Steinman. 1990. Dendritic cells are the principal cell in mouse spleen bearing immunogenic fragments of foreign proteins. J. Exp. Med. 172:383.

9. Hagerty, D. T., B. D. Evavold, and P. M. Allen. 1991. The processing and presentation of the self-antigen hemoglobin. J. Immunol. 147:3282-3288.

10. Mordes, J. P., J. Desemone, and A. A. Rossini. 1987. The BB rat. Diabetes/Metab. Rev. 3:725-750.

11. Parfrey, N. A., G. J. Prud'homme, E. Colle, A. Fuks, T. A. Seemayer, and R. D. Guttmann. 1989. Immunologic and genetic studies of diabetes in the BB rat. CRC Crit. Rev. Immunol. 9:45-65.

12. Crisá, L., J. P. Mordes, and A. A. Rossini. 1992. Autoimmune diabetes mellitus in the BB rat. Diabetes/Metab. Rev. 8:9-37.

13. Hanenberg, H., V. Kolb-Bachofen, G. Kantwerk Funke, and H. Kolb. 1989. Macrophage infiltration precedes and is a prerequisite for lymphocytic insulitis in pancreatic islets of pre-diabetic BB rats. Diabetologia. 32:126-134.

14. Kolb, H., G. Kantwerk, U. Treichel, T. Kurner, U. Kiesel, T. Hoppe, and V. Kolb-Bachofen. 1986. Prospective analysis of islet lesions in BB rats. Diabetologia. 29:559A. (Abstr.)

15. Lee, K. U., M. K. Kim, K. Amano, C. Y. Pak, M. A. Jaworski, J. G. Mehta, and J.-W. Yoon. 1988. Preferential infiltration of macrophages during early stages of insulitis in diabetes-prone BB rats. Diabetes. 37:1053-1058.

16. Voorbij, H. A. M., P. H. M. Jeucken, P. J. Kabel, M. de Haan, and H. A. Drexhage. 1989. Dendritic cells and scavenger macrophages in pancreatic islets of prediabetic BB rats. Diabetes. 38:1623-1629.

17. Like, A. A., E. Kislauskis, R. M. Williams, and A. A. Rossini. 1982. 
Neonatal thymectomy prevents spontaneous diabetes mellitus in the BB/W rat. Science (Wash. DC). 216:644-646.

18. Like, A. A., C. A. Biron, E. J. Weringer, K. Byman, E. Sroczynski, and D. L. Guberski. 1986. Prevention of diabetes in BioBreeding/Worcester rats with monoclonal antibodies that recognize $T$ lymphocytes or natural killer cells. $J$. Exp. Med. 164:1145-1159.

19. Oschilewski, U., U. Kiesel, and H. Kolb. 1985. Administration of silica prevents diabetes in BB-rats. Diabetes. 34:197-199.

20. Koevary, S., A. A. Rossini, W. Stoller, W. Chick, and R. M. Williams. 1983. Passive transfer of diabetes in the BB/W rat. Science (Wash. DC). 220:727-728.

21. McKeever, U., J. P. Mordes, D. L. Greiner, M. C. Appel, J. Rozing, E. S. Handler, and A. A. Rossini. 1990. Adoptive transfer of autoimmune diabetes and thyroiditis to athymic rats. Proc. Natl. Acad. Sci. USA. 87:7718-7722.

22. Butler, L., D. L. Guberski, and A. A. Like. 1988. Genetics of diabetes production in the Worcester colony of the BB rat. In Frontiers in Diabetes Research: Lessons from Animal Diabetes II. E. Shafrir and A. E. Renold, editors. John Libbey, London. 74-78.

23. Like, A. A., D. L. Guberski, and L. Butler. 1991. Influence of environmental viral agents on frequency and tempo of diabetes mellitus in BB/Wor rats. Diabetes. 40:259-262.

24. Bowers, W. E., M. S. Ruhoff, E. M. Goodell, and J. K. Stoltenborg. 1988. The effect of silica treatment on accessory cell-dependent rat $\mathrm{T}$ lymphocyte proliferation. Immunobiology. 176:179-194.

25. Inaba, K., J. P. Metlay, M. T. Crowley, and R. M. Steinman. 1990. Dendritic cells pulsed with protein antigens in vitro can prime antigen-specific, MHCrestricted T cells in situ. J. Exp. Med. 172:631-640.

26. Bowers, W. E., M. S. Ruhoff, and E. M. Goodell. 1990. Conditioned medium from activated rat macrophages and the recombinant factors, IL-1 beta and GM-CSF, enhance the accessory activity of dendritic cells. Immunobiology. 180:362-384.

27. Klinkert, W. E. F., J. H. LaBadie, J. P. O'Brien, C. F. Beyer, and W. E. Bowers. 1980. Rat dendritic cells function as accessory cells and control the production of a soluble factor required for mitogenic responses of $\mathrm{T}$ lymphocytes. Proc. Natl. Acad. Sci. USA. 77:5414-5418.

28. Levitt, D., and R. Danen. 1986. Separation of lymphocyte subpopulations using biotin-avidin erythrocyte rosettes. J. Immunol. Methods. 89:207-211.

29. Beyer, C. F., and W. E. Bowers. 1977. Lymphocyte transformation induced by chemical modification on membrane components. I. Characteristics of the direct and indirect restimulation responses of rat lymph node cells to periodate. J. Immunol. 119:2120-2128.

30. Bowers, W. E., and E. M. Goodell. 1984. Dendritic cells: historical perspective and role in oxidative mitogenesis. Immunobiology. 168:274-284.

31. Gilman, S. C., B. A. Woda, and J. D. Feldman. 1981. T lymphocytes of young and aged rats. I. Distribution, density and capping of T antigens. J. Immunol. 127:149-153.
32. Greiner, D. L., E. S. Handler, K. Nakano, J. P. Mordes, and A. A. Rossini. 1986. Absence of the RT-6 T cell subset in diabetes-prone BB/W rats. $J$. Im munol. 136:148-151.

33. Nie, N. H., C. H. Hull, J. G. Jenkins, K. Steinbrenner, and D. H. Bent 1975. Statistical Package for the Social Sciences. McGraw-Hill, Inc., New York.

34. Koide, S. L., K. Inaba, and R. M. Steinman. 1987. Interleukin-1 enhances T-dependent immune responses by amplifying the function of dendritic cells. $J$ Exp. Med. 165:515-530.

35. Rochester, C. L., E. M. Goodell, J. K. Stoltenborg, and W. E. Bowers. 1988. Dendritic cells from rat lung are potent accessory cells. Am. Rev. Respir. Dis. 138:121-128.

36. Guberski, D. L., L. Butler, W. Kastern, and A. A. Like. 1989. Genetic studies in inbred BB/Wor rats: Analysis of progeny produced by crossing lymphopenic diabetes-prone rats with nonlymphopenic diabetic rats. Diabetes. 38:887-893.

37. Klinkert, W. E. F. 1990. Lymphoid dendritic accessory cells of the rat. Immunol. Rev. 117:103-120.

38. Bowers, W. E., and M. R. Berkowitz. 1986. Differentiation of dendritic cells in cultures of rat bone marrow cells. J. Exp. Med. 163:872-883.

39. Fossum, S. 1991. Dendritic leukocytes: features of their in vivo physiology. Res. Immunol. 140:877-926.

40. Rothe, H., K. Fehsel, and H. Kolb. 1990. Tumour necrosis factor alpha production is upregulated in diabetes prone BB rats. Diabetologia. 33:573-575.

41. Prud'homme, G. J., A. Fuks, E. Colle, T. A. Seemayer, and R. D. Guttmann. 1984. Immune dysfunction in diabetes-prone BB rats: interleukin 2 production and other mitogen-induced responses are suppressed by activated macrophages. J. Exp. Med. 159:463-478.

42. Woda, B. A., and C. Padden. 1986. Mitogen responsiveness of lymphocytes from the BB/W rat. Diabetes. 35:513-516.

43. Voorby, H. A. M., P. J. Kabel, M. de Haan, P. H. M. Jeucken, R. D. Van der Gaag, M. H. De Baets, and H. A. Drexhage. 1990. Dendritic cells and class II MHC expression on thyrocytes during the autoimmune thyroid disease of the BB rat. Clin. Immunol. Immunopathol. 55:9-22.

44. Georgiou, H. M., and D. Bellgrau. 1989. Thymus transplantation and disease prevention in the diabetes-prone Bio-Breeding rat. J. Immunol. 142:3400-3405.

45. Clare-Salzler, M., J. Brooks, A. Chai, K. Van Herle, and C. Anderson. 1991. In vivo effects of pancreatic lymph node dendritic cells in NOD mice. Diabetes. 40(Suppl. 1):52A. (Abstr.)

46. Grubeck-Loebenstein, B., G. Buchan, D. Chantry, H. Kassal, M. Londei, K. Pirich, M. Turner, W. Walshausl, and M. Feldmann. 1989. Analysis of intrathyroidal cytokine production in thyroid autoimmune disease: thyroid follicular cells produce interleukin-1 alpha and interleukin-6. Clin. Exp. Immunol. 77:324.

47. Cavallo, M. G., M. G. Baroni, A. Toto, A. J. H. Gearing, T. Forsey, D. Andreani, R. Thorpe, and P. Pozzilli. 1992. Viral infection induces cytokine release by beta islet cells. Immunology. 75:664-668. 